\title{
Obtenção experimental do coeficiente de arrasto com o lançamento de fo- guetes de garrafas PET $^{+*}$
}

Jessica de Oliveira Chang

Felipe Luiz Quinalha Costa ${ }^{1}$

Iago Cipriano Dutra ${ }^{l}$

Gabrielle Aquino Ferreira Nery ${ }^{1}$

Luis Henrique Nery ${ }^{1}$

Emilly Rafaelly Rabelo Rodrigues ${ }^{1}$

Angelo Melari Garcia Selin ${ }^{1}$

Felipe Teixeira Zambello ${ }^{1}$

Estudantes da Universidade Federal de Alfenas - Iniciação Científica

Daniel Juliano Pamplona da Silva ${ }^{l}$

Rodrigo Rocha Cuzinatto ${ }^{1}$

Instituto de Ciência e Tecnologia - Universidade Federal de Alfenas

Poços de Caldas - MG

\section{Resumo}

$O$ objetivo deste artigo é medir o coeficiente de arrasto $k$ do ar para baixas velocidades de foguetes de garrafa PET propelidos a água e ar comprimido. Fazemos isso através de dois tipos de movimento, a saber, vertical (queda) e lançamento oblíquo. Encontramos os valores $k=$ $(0,05 \pm 0,02) s^{-1}$ e $k=(0,03 \pm 0,02) s^{-1}$ para os movimentos vertical e oblíquo respectivamente.

Palavras-chave: Coeficiente de Arrasto; Foguetes de Garrafa PET; Movimentos Vertical e Oblíquo.

\footnotetext{
${ }^{+}$Experimental determination of the drag-force coefficient through soda-bottle rocket launching

* Recebido: dezembro de 2018. Aceito: julho de 2019.

1 E-mails: top897.jc@gmail.com; felipequinalha97@gmail.com; iagosouzaecia@ gmail.com; nery98.aquino@gmail.com; luishqnery@gmail.com; emillyrabelo5@gmail.com; aselin55@gmail.com; felipe.zambello1@gmail.com; daniel.silva@unifal-mg.edu.br; rodrigo.cuzinatto@unifal-mg.edu.br
} 


\begin{abstract}
This paper's main objective is to measure the drag-force coefficient for low-velocity movements of pressurized-air-water soda-bottle rockets. This is performed via two types of flights, namely vertical fall and inclined launch. The determined values are $k=(0.05 \pm 0.02) s^{-1}$ for the vertical fall and $k=(0.03 \pm 0.02) s^{-1}$ for the inclined motion.
\end{abstract}

Keywords: Drag-force Coefficient; Soda-bottle Rockets; Vertical Fall and Inclined Motion.

\title{
I. Introdução
}

Neste projeto é abordada a física que rege os movimentos de queda ${ }^{2}$ e lançamento oblíquo de um foguete. Para nos aproximarmos de um modelo realístico que leva em conta a resistência do ar, é inserido o termo de arrasto linear na velocidade nas equações de movimento. O lançamento de foguetes de garrafas PET foi amplamente estudado na literatura no caso dos movimentos desprezando a resistência do ar, vide e.g. as Ref. (SOUZA, 2007; CUZINATTO, 2015; CUZINATTO, 2017). O caso do movimento de foguetes artesanais com o arrasto foi abordado no estudo (QUEIROZ, 2017) apenas do ponto de vista teórico. O presente trabalho completa o estudo, aborda o problema do experimental, destaca e adapta as equações fundamentais do estudo teórico anterior e apresenta a medida de $k$ em duas montagens experimentais distintas, como veremos a seguir.

A equação de movimento com arrasto do projétil pode ser escrita de forma compacta por (MARION, 1995) ${ }^{3}$ :

$$
m \frac{d \vec{v}}{d t}=-m k \vec{v}+\vec{P}
$$

sendo $\vec{v}=v_{x} \hat{\imath}+v_{y} \hat{\jmath}$ a velocidade do projétil (HALLIDAY, 2008), $t$ o tempo, $k$ o coeficiente de arrasto e $\vec{P}=-m g \hat{\jmath}$ é a força peso, em que $m$ é a massa e $g$ é a magnitude da aceleração gravitacional. Nota-se que o termo de arrasto $\left(\overrightarrow{F_{r}}=-k m \vec{v}\right)$ é assumido como linear com a velocidade no regime de baixas velocidades. Esse termo é sempre negativo, pois o arrasto é contrário à tendência de movimento.

\footnotetext{
2 Usaremos o termo "queda" para descrever o movimento vertical descendente com arrasto.

3 A referência (FINNEY, 2000) aborda o problema do movimento de foguetes na presença de arrasto levando em conta um termo quadrático na velocidade. A propósito, essa é a modelagem mais comum na literatura, e.g. (HALIDAY, 2008) e (RIVEROS, 2016). Entretanto, como enfatizado em (MARION, 1995), essa estratégia tem aplicabilidade mais adequada a um regime de velocidades altas (maiores ou da ordem de $24 \mathrm{~m} / \mathrm{s}$ ) e, portanto, alheio à situação experimental deste artigo, conforme discutido na nossa seção 4.
} 
A equação diferencial (1) pode ser resolvida componente a componente, usando $v_{x}=d x / d t$ e $v_{y}=d y / d t$, sendo $x$ e $y$ as componentes horizontal e vertical usuais respectivamente. A solução é:

$$
\begin{gathered}
\frac{d x}{d t}=v_{0 x} e^{-k t} \\
\frac{d y}{d t}=-\frac{g}{k}+\left(\frac{g}{k}+v_{0 y}\right) e^{-k t},
\end{gathered}
$$

onde $v_{0}$ é a velocidade inicial do projétil, $v_{0 x}=v_{0} \cos \theta$ e $v_{0 y}=v_{0} \operatorname{sen} \theta$ são suas componentes nas direções $x$ e $y$, respectivamente, e $\theta$ é o ângulo com a horizontal. Após nova integração com respeito a $t$, as Eqs. (2) e (3) resultam:

$$
\begin{gathered}
x=x_{0}+\frac{v_{0 x}}{k}\left(1-e^{-k t}\right), \\
y=y_{0}-\frac{g t}{k}+\frac{1}{k}\left(v_{0 y}+\frac{g}{k}\right)\left(1-e^{-k t}\right) .
\end{gathered}
$$

É possível inserir dados experimentais em (4) e (5) para encontrar o valor do coeficiente de arrasto $k$. Neste trabalho, os projéteis usados para a coleta dos dados foram foguetes de garrafas PET pressurizadas.

$\mathrm{Na}$ próxima seção, apresentaremos as estratégias experimentais usadas para obter o valor de $k$ para movimentos a baixas velocidades de forma explícita.

A referência (WIDMARK, 1998) ocupa-se da estimativa do coeficiente de arrasto no regime de altas velocidades, em que a força de arrasto é proporcional ao quadrado da velocidade do foguete. O preço a pagar por essa escolha é a sofisticação da técnica experimental requerida. Com efeito, o autor dessa referência usa túnel de vento para coletar dados que alimentam um software para produzir a estimativa do coeficiente desejado. Nosso trabalho prescinde dessa sofisticação e é um método inteiramente analítico corroborado por uma estimativa numérica (vide seção 4).

\section{Metodologia}

Foram realizadas duas formas distintas para a obtenção de dados, sendo a primeira a queda livre, e a segunda o lançamento oblíquo.

Para ambos os experimentos, foram utilizados foguetes construídos a partir de garrafas PET de refrigerantes de 1,5 L levando-se em conta seu centro de massa (SOUZA, 2007), aerodinâmica e reprodutibilidade.

Na construção dos foguetes, as garrafas PET foram utilizadas como estrutura principal acoplando a elas aletas de isopor, fixadas por cola quente e fitas adesivas. No bico do fo- 
guete foi colocado um material de maior densidade (conjunto parafuso e porca) com intuito de deslocar o centro de massa em direção à ogiva do foguete, aumentando a estabilidade do seu movimento. Roteiros detalhados de construção dos foguetes e de suas bases de lançamento podem ser encontrados em outros trabalhos na literatura, vide e.g. (SOUZA, 2007; CUZINATTO, 2015; CUZINATTO, 2017). Aqui, a ênfase é o estudo pormenorizado do movimento dos projeteis.

As estratégias empregadas na obtenção de dados a partir dos movimentos vertical e oblíquo dos foguetes possuem suas respectivas peculiaridades. As próximas duas seções buscam esclarecer e detalhar os diferentes casos.

\section{II.1 Queda}

Primeiramente, escolhemos o ponto mais alto e acessível disponível no local de realização do experimento (Instituto de Ciência e Tecnologia - Campus Poços de Caldas). Como cada foguete foi usado para cerca de dez medidas, usaram-se colchonetes no local de impacto para se evitar que os choques danificassem a estrutura dos protótipos (Fig. 1(a)).

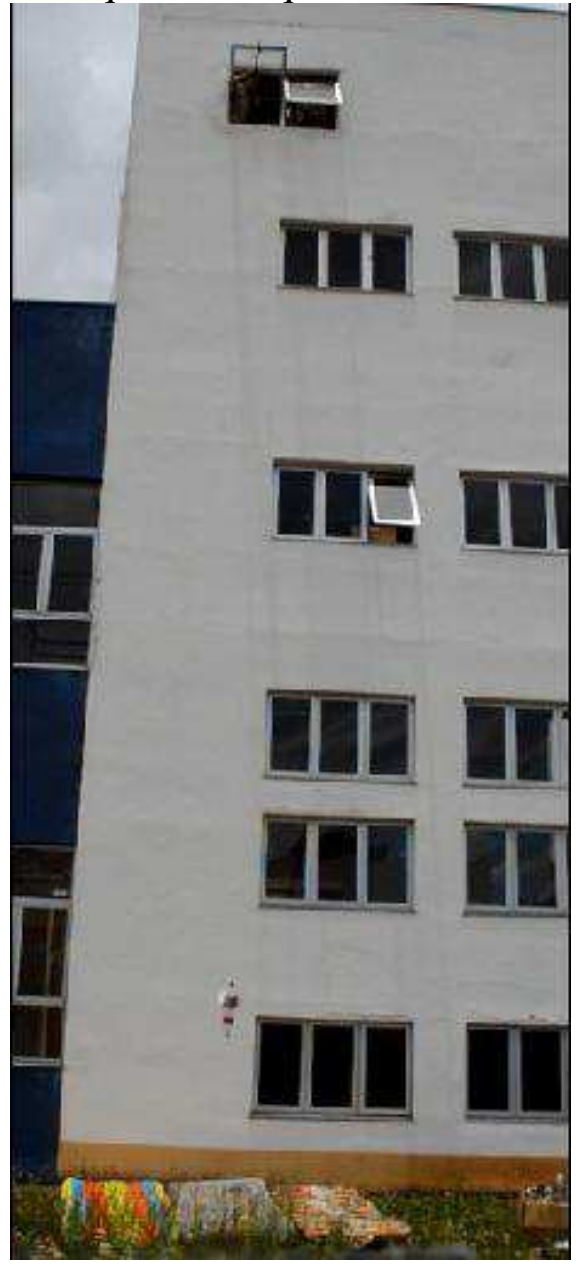

(a)

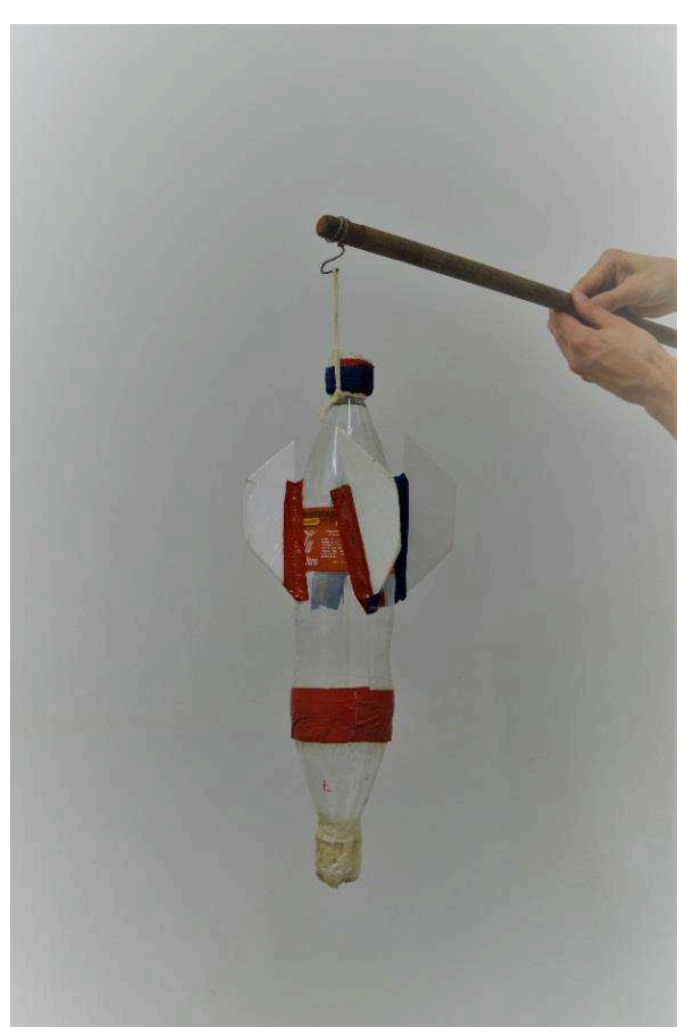

(b)

Fig. 1 - (a) Instantâneo de uma das quedas do foguete PET. (b) Gancho utilizado para o abandono de cada protótipo e detalhe do foguete de garrafa PET. 
Com o auxílio de uma trena de 30 m e menor divisão 2 mm, mediu-se a distância entre o ponto em que o foguete inicia sua trajetória de queda e a superfície superior dos colchonetes (Fig. 1(a)). Além disso, foi subtraído da distância de queda (altura) o comprimento do foguete, resultando na distância que a ponta do foguete percorreu. Utilizamos um gancho em formato de anzol na extremidade de um cabo de vassoura (Fig. 1(b)) para prender a parte inferior do foguete a partir de um laço de barbante. Girando o gancho de $180^{\circ}$, garantimos que os foguetes eram abandonados do repouso em todas as repetições. O cabo de vassoura era sempre apoiado na superfície horizontal plana da janela do topo do edifício, cf. Fig. 1(a).

O caminho percorrido pelos foguetes ao longo da queda foi registrado com o auxílio de uma câmera fotográfica de 50 fps. O posicionamento desta foi tal que toda a trajetória de cada foguete pôde ser devidamente registrada, desde seu desprendimento do gancho até o contato com o colchonete. Os foguetes eram içados por uma corda ao topo do prédio a partir de suas alças de barbante entre os lançamentos.

As medidas dos tempos de voo obtidas a partir da filmagem da queda livre foram analisadas em câmera lenta com um programa de edição de vídeos (Windows Movie Maker), sendo a velocidade entre os frames diminuída em oito vezes. Isso foi usado na estimativa da incerteza do tempo de queda.

A incerteza na medição do tempo $\sigma_{t}$ foi majorada como a soma direta da incerteza do software de tratamento de vídeo $(0,04 s)$ e a incerteza da câmera de filmagem $(0,02 s)$. O valor é $\sigma_{t}=0,06 \mathrm{~s}$; essa estimativa foi usada tanto no estudo da queda, abordado nesta seção, quanto no do lançamento oblíquo, estudado na seção a seguir.

\section{II.2 Lançamento oblíquo}

Diferentemente da queda, os lançamentos oblíquos fizeram uso de uma base lançadora construída para este projeto que contava com um manômetro capaz de indicar a pressão dentro dos foguetes, uma bomba de ar manual e dois compressores automotivos portáteis de $12 \mathrm{~V}$, acoplados à bateria de um veículo para pressurizar os foguetes. A Fig. 2 mostra a montagem foguete-base. A bomba e os compressores são acoplados a base através de bicos de pneu fixados em niples de PVC.

São utilizados canos de PVC de $3 / 4$ " para suporte e estabilidade da base. Canos de $1 / 2$ " acoplam o foguete à base e servem de canal de injeção de ar no sistema. Por questões de segurança, uma válvula de escape (VE) - haste azul na Fig. 2(c) - está disponível para despressurizar a câmara em casos de emergência. Outros detalhes de construção constam da Ref. (CUZINATTO, 2017).

O foguete é preso à base por um sistema de lacre composto por fitas Hellerman, uma mola e um pino de segurança. Ao atingir a pressão desejada, retira-se o pino e o foguete é disparado pela diferença da pressão entre o interior do foguete e a atmosfera. 


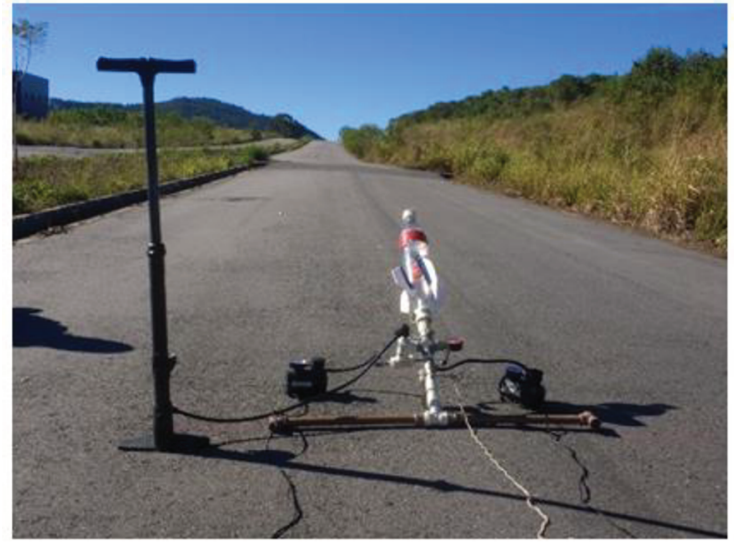

(a)

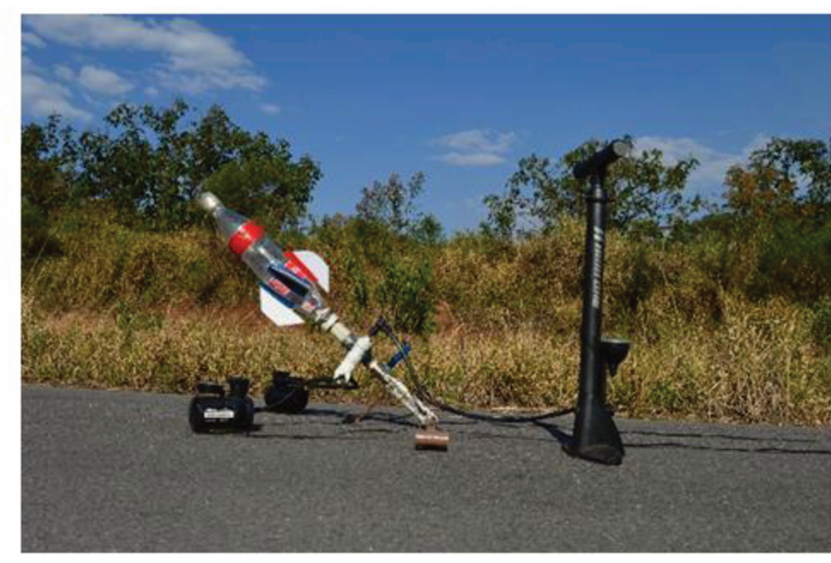

(b)

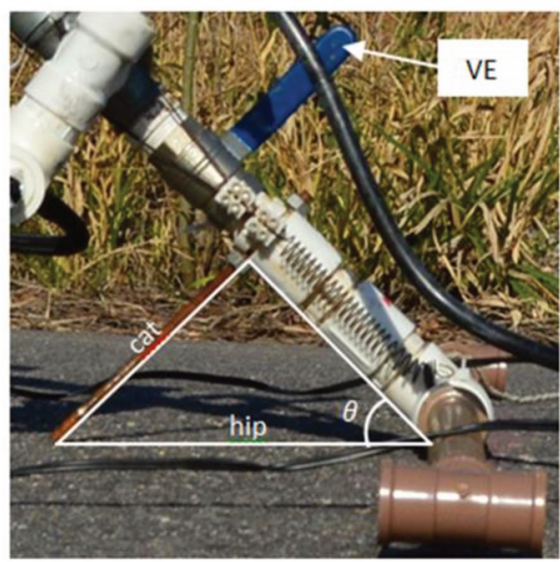

(c)

Fig. 2 - Montagem foguete-base. (a) vista traseira com perspectiva da pista de lançamento e aterrisagem; (b) vista lateral; (c) detalhe do ângulo de lançamento $\theta$.

A trajetória do foguete é longa e a tomada de vídeo do movimento completo com uma câmera estática não apresentaria definição suficiente no vídeo para determinarmos com acurácia as posições e os momentos de início e de fim da trajetória. Essa dificuldade motivounos a medir o tempo de voo a partir dos sons dos eventos: (i) liberação do foguete e (ii) impacto com o solo. A medição foi realizada com uma câmera à meia distância das posições dos eventos (i) e (ii). Como precisávamos dos picos sonoros em (i) e (ii), não utilizamos os colchonetes para amortecer o impacto em (ii). Ainda assim, os foguetes não sofriam avarias no impacto com o solo. O vídeo desta câmera, embora registre a imagem de uma parte da trajetória, foi utilizado somente para extração dos picos sonoros no software de tratamento de vídeo e a consequente aferição do tempo de voo.

Escolhemos um ângulo $\theta$ de $45^{\circ}$ com solo. Essa escolha tem dois motivos. O primeiro é: no lançamento oblíquo sem arrasto esse ângulo garante o alcance máximo. O segundo, 
que está relacionado ao primeiro, é: aumentando o alcance, diminui-se a incerteza relativa dessa medida. Experimentalmente, esse ângulo podia ser ajustado continuamente através de um parafuso rosqueado no cano de lançamento da base: rosquear o parafuso é equivalente a variar o cateto oposto (cat), ajustando $\theta$. A distância no chão entre o ponto de apoio da base e o ponto de apoio do parafuso é a hipotenusa (hip), vide Fig. 2(c).

A modelagem física deste trabalho - e também das Refs. (HALLIDAY, 2008) e (MARION, 1995) - exige que a altura do ponto $I$ de impacto com o solo seja igual à altura do ponto em que o foguete tem velocidade $v_{0}$ (Fig. 3). Essa velocidade é aquela velocidade adquirida pelo foguete depois que toda água foi ejetada. Chamamos de $l$ a altura do ponto $E$ ocupado pelo foguete quando do término da ejeção da água. Para satisfazer a condição de validade da modelagem (ponto $E$ e ponto $I$ no mesmo nível), a base deve estar a uma altura $l$ abaixo do ponto $I$. Realizamos isso experimentalmente escolhendo um terreno em aclive, onde a base de lançamento foi disposta na posição mais baixa. Para garantir que o ponto $E$ e o ponto $I$ tenham o mesmo nível, é preciso determinar a altura $l$ de ejeção da água. Fizemos isso com uma segunda câmera que filmou somente o disparo, de perfil (Fig. 2(b)), na região próxima à base, onde ocorria a ejeção da água. Durante a ejeção da água o foguete é acelerado a partir do repouso (velocidade nula no ponto $B$ da Fig. 3) até a velocidade $v_{0}$ (no ponto $E$ de altura $l$ na Fig. 3). A posição do ponto $E$ foi determinada a partir da posição do ponto de impacto $I$ com um manômetro em U, construído com uma mangueira d'agua transparente. Com o valor da altura $l$, o valor do ângulo $\theta$ de lançamento e a posição do ponto E é possível determinar a posição $B$ em que a base deve ser disposta por trigonometria.

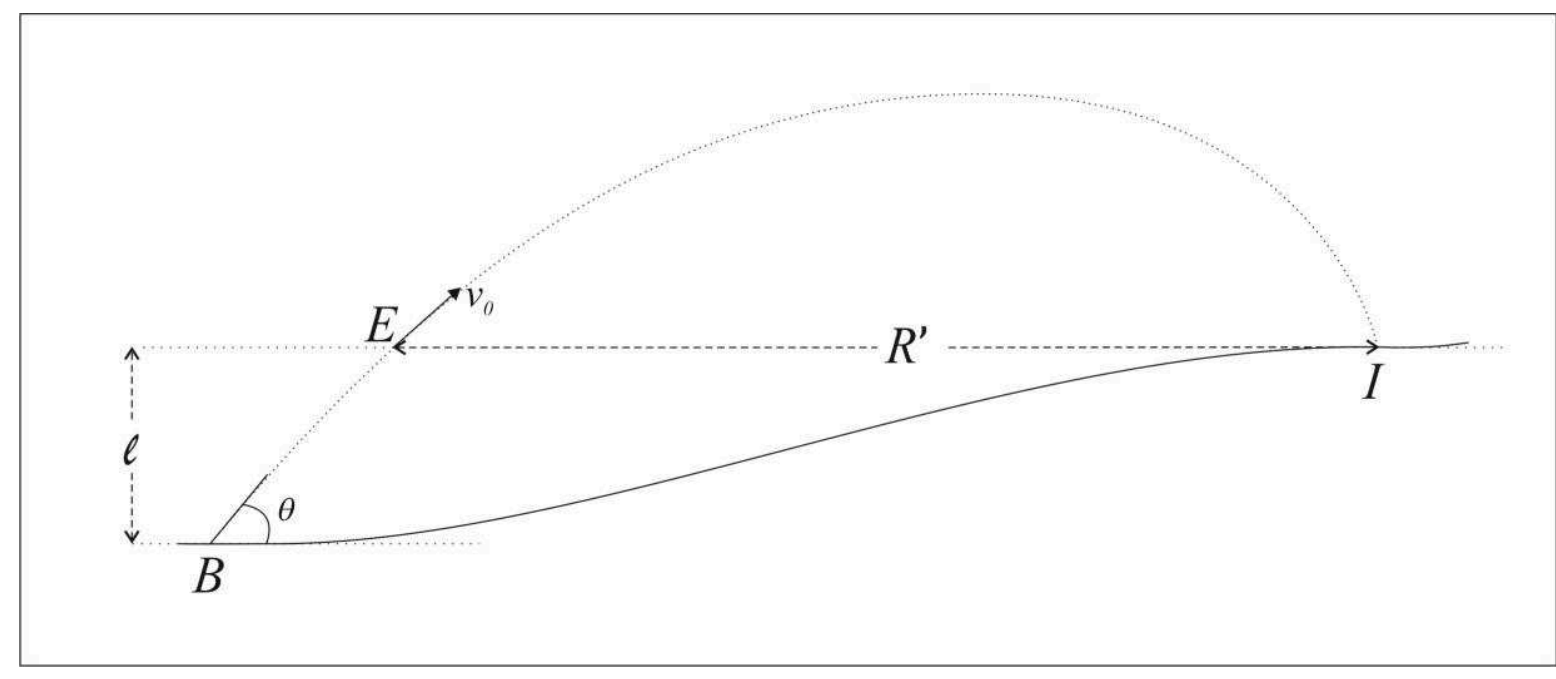

Fig. 3 - Representação de perfil do desnível no terreno de lançamento oblíquo.

\footnotetext{
${ }^{4}$ A segunda câmera usada possui especificações análogas àquela localizada no ponto médio da trajetória.
} 
Para a medida do alcance, realizávamos marcações no ponto de impacto dos foguetes com o solo. A cada lançamento fazíamos a medição da distância $d$ do ponto de impacto até a marcação de referência a 57,8 metros da base. O alcance era, então, calculado pela soma (ou subtração) de $d$ com a marcação de referência caso o foguete caísse além (aquém) da marcação. As eventuais dispersões laterais são fontes de erro na medida do alcance; esses erros foram incluídos no desvio padrão da média que são tomados como incerteza em $R^{\prime}$. A incerteza instrumental da trena $(1 \mathrm{~mm})$ é duas ordens de grandeza menor do que o desvio padrão da média $(\cong 30 \mathrm{~cm})$ e, portanto, foi desprezada.

A câmera era disposta a meia distância entre o ponto de lançamento e a distância de referência $(57,8 \mathrm{~m})$. Deste modo é possível desconsiderar a influência do tempo de propagação do som na medida do tempo de voo.

Começávamos uma nova gravação segundos antes de o foguete desprender-se de sua base. O tempo de voo em cada lançamento foi aferido analisando a faixa de áudio de sua gravação: é o intervalo entre o pico sonoro de liberação do foguete e o pico sonoro de seu impacto com o solo.

Foi realizada também a medida do tempo de aceleração do foguete (ejeção da água). Devido ao resultado ser da mesma ordem de grandeza da incerteza do tempo total de voo, a variável tempo de aceleração também foi desprezada. Como medida compensatória aumentou-se proporcionalmente a magnitude do valor da incerteza do tempo de voo.

\section{Resultados}

Nesta seção apresentamos os dados extraídos com os experimentos de queda (seção 3.1) e lançamento oblíquo (seção 3.2), descrevemos os cálculos de $k$ com base na fundamentação teórica da Introdução e detalhamos as estimativas de incerteza envolvidas. O roteiro passa pela determinação do valor do módulo da aceleração gravitacional local $g$; da expansão das expressões (4) e (5) em série de Taylor em torno de $k=0$ e pela construção a expressão analítica de $k$ com a primeira ordem de correção como função dos dados coletados. Por fim, comparamos o valor experimental de $k$ encontrado através dessas expansões com o valor de $k$ advindo da solução numérica das Eqs. (4) e (5).

Começamos pelo cálculo de $g$. O módulo da aceleração gravitacional local é determinado por (MORITZ, 2000):

$$
g=g_{e} \frac{1+\kappa \sin ^{2} \Phi}{\sqrt{1-\mathrm{e}^{2} \sin ^{2} \Phi}}
$$

sendo que $g_{e}=9,7803267715 \mathrm{~m} / \mathrm{s}^{2}$ é o módulo da gravidade normal no equador; $\mathrm{e}^{2}=$ 0,00669438002290 é a primeira excentricidade e $\kappa=0,001931851353$ é uma quantidade

\footnotetext{
${ }^{5}$ A marcação de referência foi assumida como o valor médio do alcance de três lançamentos preliminares.
} 
adimensional dependente dos valores do semieixo maior e menor da Terra. $\Phi$ é a latitude geográfica em radianos.

A latitude no local de realização do experimento $\Phi=-(21,819 \pm 0,003)^{\circ}$ foi determinada usando o software Google Earth, que pode ser acessado gratuitamente no sitio $<$ https://www.google.com.br/intl/pt-BR/earth/>. A incerteza na latitude $\Phi$ foi estimada como sendo a diferença entre as latitudes dos extremos de toda extensão dos múltiplos lançamentos - de queda e oblíquo, abarcando os locais nas Fig. 1(a) e 2(a).

Assim, com a Eq. (6), calculamos $g=(9,787463 \pm 0,000002) m / s^{2}$ (MORITZ, 2000).

\section{III.1 Queda}

Relacionamos a altura de queda $y=h$ com o tempo de voo $t=T$ e o coeficiente de arrasto $k$ com a Eq. (5). As medições descritas na seção 2.1 fornecem valores de $h$ e T. A primeira ideia seria substituir os valores do par $(h, T)$ em (5) e encontrar diretamente $k$. Porém, a Eq. (5) é transcendental em $k$ (o coeficiente $k$ aparece nos denominadores do lado direito e também como argumento de uma exponencial).

Uma solução $k=k(h, T)$ analítica, porém aproximada, é encontrada assumindo $k$ pequeno e usando a técnica de expansão em série de Taylor (MARION, 1995). 6 Expandindo, então, a equação resultante até primeira ordem de correção em $k$, chegamos à:

$$
k=\frac{6}{g T^{3}}\left(\frac{g T^{2}}{2}-h\right) .
$$

Os valores de $h$ e $T$ que alimentam a Eq. (7) são aqueles na Tabela 1. Tratam-se dos valores médios do conjunto de dados produzido nas 113 quedas de foguetes PET. As incertezas $\sigma_{h}$ e $\sigma_{T}$ em $h$ e $T$, respectivamente, são obtidas considerando-se o erro instrumental e o erro estatístico. A incerteza do valor calculado de $k$ é encontrado pelo método de propagação de incertezas (VUOLO, 1996).

Tabela 1 - Dados experimentais da queda.

\begin{tabular}{cc}
\hline Grandeza & Valor \\
\hline$\left(\boldsymbol{h} \pm \boldsymbol{\sigma}_{\boldsymbol{h}}\right) \boldsymbol{m}$ & $11,15 \pm 0,05$ \\
$\left(\boldsymbol{T} \pm \boldsymbol{\sigma}_{T}\right) \boldsymbol{s}$ & $1,529 \pm 0,008$ \\
$\left(\boldsymbol{k} \pm \boldsymbol{\sigma}_{\boldsymbol{k}}\right) \boldsymbol{s}^{-\mathbf{1}}$ & $0,05 \pm 0,02$ \\
\hline
\end{tabular}

\footnotetext{
${ }^{6}$ Em um trabalho análogo, KRAFF (2015) trata da solução analítica para o problema teórico da ascensão vertical na presença de arrasto linear com a velocidade, em um campo gravitacional constante, para um sistema de massa variável. Em nosso trabalho, estudamos a queda vertical de um foguete vazio de massa fixa e a situação experimental do movimento oblíquo com arrasto linear na velocidade depois da ejeção da água do foguete.
} 
Uma alternativa à expansão que leva a expressão analítica (7) é resolver a Eq. (5) de forma numérica para $k=k(h, T)$. Fizemos isso pelo método da bisseção e obtivemos o valor $k=(0,0496 \pm 0,0001) s^{-1}$. Esse valor é compatível com o da Tabela 1 tendo-se em vista a incerteza do valor experimental e também a precisão numérica de quatro casas decimais do caso numérico.

\section{III.2 Lançamento oblíquo}

No caso do lançamento oblíquo é necessário combinar as Eqs. (4) e (5) para obtenção do coeficiente de arrasto $k$. Isso é assim pois medimos o tempo de voo $t=T^{\prime}$ e o alcance horizontal $x=R^{\prime}$. Tanto quanto no caso anterior, precisamos fazer a expansão em série de Taylor das exponenciais para lidar com equações transcendentais em $k$. O resultado desse procedimento fornece indiretamente uma equação $k^{\prime}=k^{\prime}\left(R^{\prime}, T^{\prime}\right)$. Veja:

$$
k^{\prime}=\frac{3 g}{v_{0} \sin \theta}\left(1-\frac{g T^{\prime}}{2 v_{0} \sin \theta}\right),
$$

onde $v_{0}=v_{0}\left(R^{\prime}, T^{\prime}\right)$ é dado pela expressão:

$$
v_{0}=\frac{g T^{\prime}}{3 \sin \theta}\left(1+\sqrt{1-\frac{3 R^{\prime} \tan \theta}{2 g T^{\prime 2}}}\right) .
$$

Aqui, usamos a notação com linha ( ' ) para diferenciar os resultados obtidos no lançamento oblíquo dos resultados da queda livre. Os valores de $T^{\prime}$ e $R^{\prime}$ empregados nas Eqs. (8) e (9) constam da Tabela 2 e são o resultado do tratamento de dados relativos aos 122 lançamentos realizados. Note que a incerteza $\sigma_{T^{\prime}}$ do tempo de voo no caso oblíquo (Tabela 2) é uma ordem de grandeza maior que a incerteza $\sigma_{T}$ para o tempo de voo da queda (Tabela 1). A origem dessa diferença é a dispersão no conjunto das medidas de tempo: ela é maior para o caso oblíquo do que para a queda. ${ }^{7}$ A dispersão afeta o desvio padrão, que é um dos componentes da incerteza da média juntamente com a incerteza instrumental e o número de medidas (VUOLO, 1996).

Com as Eq. (8) e (9) é possível deduzir a fórmula para determinar a incerteza da velocidade e da constante de arrasto usando, novamente, a técnica de propagação de erros (VUOLO, 1996). Os valores de $k^{\prime}, v_{0}$ e suas respectivas incertezas aparecem na Tabela 2.

\footnotetext{
${ }^{7}$ As condições de reprodutibilidade do lançamento oblíquo são bem piores do que na queda.
} 
Tabela 2 - Dados experimentais do lançamento oblíquo.

\begin{tabular}{cc}
\hline Grandeza & Valor \\
\hline$\left(\boldsymbol{\theta} \pm \boldsymbol{\sigma}_{\boldsymbol{o}}\right)$ & $0,785 \pm 0,001$ \\
$\left(\boldsymbol{v}_{\boldsymbol{o}} \pm \boldsymbol{\sigma}_{\boldsymbol{v}_{\boldsymbol{o}}}\right) \boldsymbol{m} / \boldsymbol{s}$ & $22,5 \pm 0,2$ \\
$\left(\boldsymbol{R}^{\prime} \pm \boldsymbol{\sigma}_{\boldsymbol{R}^{\prime}}\right) \boldsymbol{m}$ & $48,0 \pm 0,3$ \\
$\left(\boldsymbol{T}^{\prime} \pm \boldsymbol{\sigma}_{\boldsymbol{T}^{\prime}}\right) \boldsymbol{s}$ & $3,19 \pm 0,02$ \\
$\left(\boldsymbol{k}^{\prime} \pm \boldsymbol{\sigma}_{\boldsymbol{k}^{\prime}}\right) \boldsymbol{s}^{-\mathbf{1}}$ & $0,03 \pm 0,02$ \\
\hline
\end{tabular}

O valor de $k^{\prime}$ pelo método numérico da bisseção é $(0,0344 \pm 0,0001) s^{-1}$. A compatibilidade desse resultado com o valor experimental de $k^{\prime}$ na Tabela 2 mostra que a expansão em Taylor é perfeitamente razoável como método analítico de obtenção do coeficiente de arrasto (assim como já havíamos observado no experimento de queda da seção anterior).

\section{Discussão e conclusão}

Neste trabalho estudamos a determinação experimental do coeficiente de arrasto de foguetes de garrafas PET em movimento no ar. Os tipos de movimento foram dois: queda (vertical) e lançamento oblíquo.

Na queda, os foguetes foram abandonados (com $\left.v_{0}=0\right)$ do alto de um prédio; medimos a altura de queda $h$ e o tempo de voo $T$. No lançamento oblíquo, usamos uma base lançadora de tubos de PVC com um sistema de pressurização que conferia velocidade inicial $v_{0} \neq 0$ em uma angulação $\theta=45^{\circ}$; medimos o alcance $R^{\prime}$ e o tempo de voo $T^{\prime}$.

Com esses dados e as Eqs. (7), (8) e (9), foram determinados os valores para o coeficiente de arrasto na queda $(k)$ e no lançamento oblíquo $\left(k^{\prime}\right)$ como $k=(0,05 \pm 0,02) s^{-1}$ e $k^{\prime}=(0,03 \pm 0,02) s^{-1}$. Comparando esses valores, observa-se que esses resultados são compatíveis dentro do intervalo de confiança de $1 \sigma$. Com isso, pode-se dizer que o valor verdadeiro de $k$ está necessariamente dentro do intervalo de $0,03 s^{-1}$ a $0,07 s^{-1}$, enquanto o valor verdadeiro de $k^{\prime}$ está no intervalo de $0,01 s^{-1}$ a $0,05 s^{-1}$. Analisando a intersecção desses intervalos, nota-se que o valor verdadeiro pode ser comum aos dois casos. Isso é esperado já que o coeficiente de arrasto deve depender das características do projétil e do fluido dentro do qual ele se move. Veja, por exemplo, a modelagem dada para $k$ na referência (HALLIDAY, 2008), onde o coeficiente de arrasto depende da densidade do fluido e da seção reta do corpo em movimento. Em nossos experimentos, o fluido, o projétil são os mesmos tanto na queda quanto no lançamento oblíquo, por isso espera-se que $k$ e $k^{\prime}$ assumam os mesmos valores.

Encontramos também os valores de $k$ e $k^{\prime}$ resolvendo numericamente as equações (4) e (5), que são transcendentais no coeficiente de arrasto. Os valores encontrados, $k=$ 
$(0,0496 \pm 0,0001) s^{-1}$ e $k^{\prime}=(0,0344 \pm 0,0001) s^{-1}$, satisfazem a condição de $k$ pequeno. Por outro lado, $k$ e $k^{\prime}$ experimentais são compatíveis com os valores numéricos. Isso justifica a validade da hipótese $k \ll 1$ necessária para a expansão em Taylor que fizemos anteriormente. Ademais, $k$ e $k^{\prime}$ são notadamente diferentes de zero em ambos os casos (dentro das incertezas e da precisão numérica), o que corrobora a existência do arrasto.

Podemos também destacar o valor da velocidade inicial no lançamento oblíquo: $v_{0}=(81,0 \pm 0,7) \mathrm{km} / \mathrm{h}$, assim como o valor final da velocidade com que o foguete colide com o solo no caso da queda vertical: $v=(51,8 \pm 0,8) \mathrm{km} / \mathrm{h}$. Esses são os valores máximos de velocidade desenvolvida pelos foguetes e, ainda assim, são menores do que $24 \mathrm{~m} / \mathrm{s}=$ $86,4 \mathrm{~km} / \mathrm{h}$; este valor é estabelecido na Ref. (MARION, 1995) como o limite de validade para a aproximação linear da força de arrasto como função da velocidade. Isso garante a adequação do modelo aos experimentos realizados e descritos aqui.

Note que $v_{0}$ do lançamento oblíquo é relativamente próximo do limite de $86,4 \mathrm{~km} / \mathrm{h}$ (a diferença é igual a $7 \sigma_{v_{o}}$ ). Isso pode contribuir para a incerteza elevada (da mesma ordem de grandeza) do valor do coeficiente de arrasto. Entretanto, justificamos a escolha desse valor de $v_{0}$ para a realização do experimento assim: um valor alto de $v_{0}$ maximiza o alcance $R^{\prime}$, diminuindo o erro relativo nessa medida.

Verifica-se que a velocidade inicial do lançamento oblíquo e a velocidade final da queda são da mesma ordem de grandeza, assim como suas incertezas, mostrando consistência no método utilizado, de acordo com o parágrafo anterior. $\mathrm{O}$ valor da velocidade final de queda vertical com arrasto, $(51,8 \pm 0,8) \mathrm{km} / \mathrm{h}$, é distinto do valor da hipotética velocidade de queda livre $(53,852 \pm 0,025) \mathrm{km} / \mathrm{h}$ mesmo considerando suas incertezas. Isso aumenta a confiança que temos na consistência dos resultados para $k$.

Seguem alguns comentários a respeito do movimento do foguete durante a ejeção da água no lançamento oblíquo. A nossa filmagem mostrou que o tempo de ejeção foi de $(0,085 \pm 0,007) s$ e nossos cálculos mostraram que a velocidade $v_{0}$ ao final da ejeção era $(22,46 \pm 0,23) \mathrm{m} / \mathrm{s}$. Usamos uma pressurização de 70 psi no foguete. O trabalho (SOUZA, 2007) faz um estudo detalhado do movimento do foguete durante a ejeção da água para um lançamento vertical, no qual é usado $80 p s i$ para pressurização na câmara do foguete. Os resultados obtidos naquele trabalho para uma quantidade de água de $600 \mathrm{ml}$ dentro do foguete de $2 \mathrm{~L}$ foram $80 \mathrm{~ms}$ e $20 \mathrm{~m} / \mathrm{s}$ respectivamente para ejeção de água e velocidade $v_{0}$. Concluímos, então, que esses valores são compatíveis com os nossos, dadas as pequenas diferenças no arranjo experimental, tais como valores de pressão, tamanho e geometria dos foguetes, e o fato crucial de que aqui $k \neq 0$.

Trabalhos futuros poderiam refinar nossos resultados experimentais do coeficiente de arrasto. Esse refinamento poderia incluir uma vídeo-análise dos lançamentos (com o software Traker, por exemplo). Tal análise potencialmente viabilizaria a obtenção dos dados de posição em função do tempo, de velocidade em função do tempo e a construção dos gráficos de trajetória, os quais poderiam ser comparados com aqueles da referência (QUEIROZ, 2017). As 
dificuldades técnico-experimentais envolvidas nessas tarefas escapam ao objetivo deste trabalho. Ainda assim, este artigo torna claro que um arranjo artesanal simples pode mostrar a existência do arrasto no movimento de corpos na atmosfera.

\section{Agradecimentos}

Os autores são gratos ao Ministério da Educação do Governo Federal do Brasil (edital PROBEXT 2014 MEC/SESu) e ao Programa de Educação Tutorial (PET) -grupo PET Ciência - pelo apoio financeiro. Agradecemos aos estudantes Arthur M. D’Ambrosio, Hugo F. de Andrade, Bárbara R. Duarte, Victor C. Lorencetti, Sérgio A. Maéstri, Renan D. Martins, Alessandra M. de Queiroz e Mauro F. de Toledo Filho por colaborações anteriores que motivaram este trabalho. Somos gratos também ao estudante José Augusto de Oliveira pelas discussões na etapa inicial deste trabalho.

\section{Referências}

CUZINATTO, R. R. et al. Construindo um foguete de garrafa pet e sua base de lançamento de PVC: o protótipo Rocketeers UNIFAL-MG, Física na Escola, v. 15, p. 52-59, 2017.

CUZINATTO, R. R. et al. Rocketeers UNIFAL-MG: o ensino de física através o lançamento de foguetes artesanais, Revista Ciência em Extensão, v. 11, p. 40-62, 2015.

FINNEY, G. A. Analysis of a water-propelled rocket: A problem in honors physics, American Journal of Physics, v. 68, p. 223-227, 2000.

HALLIDAY, D.; RESNICK, R.; WALKER, J. Fundamentos de Física. 8. ed. Rio de Janeiro: LTC, 2008, v. 1.

KRAFF, A. V. et al. Obtención y solución a la ecuación de movimiento de un cohete, actuando sobre él las fuerzas externas del campo gravitacional constante y el rozamiento del aire proporcional a la velocidade. Revista Mexicana de Física E, v. 61, p. 6-10, 2015.

MARION, J. B.; THORNTON, S. T. Classical Dynamics of particles and systems, 4. ed. Orlando: Saunders College Publishing, 1995.

MORITZ, H. Geodetic reference system 1980. Journal of Geodesy, v. 74, p. 128-133, 2000.

QUEIROZ, A. M.; CUZINATTO, R. R. O efeito do arrasto no lançamento de foguetes artesanais: aspectos teóricos. Revista Brasileira de Iniciação Científica, v. 4, p. 82-109, 2017.

RIVEROS, H. G. Taller: La computadora en el Laboratorio, Latin-American Journal of Physics Education, v. 10, p. 4325-1-4325-14, 2016.

SOUZA, J. A. Um foguete de garrafas pet. Física na Escola, v. 8, p. 4-11, 2007. 
VUOLO, J. H. Fundamentos da Teoria de Erros. 2. ed. São Paulo: Edgard Blücher, 1996.

WIDMARK, S. A. Rocket Physics. The Physics Teacher, v. 36, n. 3, p. 148-153, 1998.

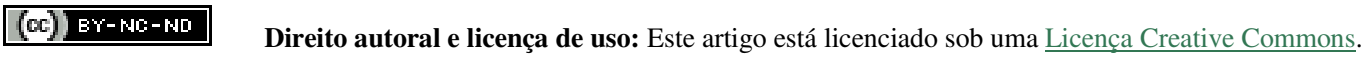

